\title{
Transfusión Intravascular Intrauterina en Isoinmunización RH
}

\author{
PRESENTACION DE UN CASO
}

\author{
Dres.: Eduardo Ortiz Herrera*, Eduardo Acosta Lleras**, Eduardo Acosta Cajiao***
}

\section{TRANSFUSION INTRAUTERINA}

A pesar del ampliamente conocido método de prevenir la isoinmunización por $\mathrm{Rh}$, por medio de la inyección oportuna del anti $\mathrm{D}$ (rhesuman), aún se presenta un escaso número de mujeres con el problema de este tipo de isoinmunización (1).

La isoinmunización puede ocurrir si no se suministra a tiempo el Anti-D a las pacientes, después de un parto, aborto, ectópico, una amniocentesis, o que hayan recibido una transfusión de sangre incompatible.

El manejo de los casos con isoinmunización severa, se ha intentado resolver con Plasmaféresis materna a repetición durante el embarazo, pero éste método ha demostrado ser costoso y con poco éxito (5). La transfusión intrauterina reportada por primera vez por Liley en 1963 (1), se ha convertido en un método adecuado y aceptado en los centros perinatales, para tratar al feto inmaduro con enfermedad hemolítica severa $(2,4)$.

El método empleado por Liley y sus seguidores, es el de transfundir glóbulos rojos empaquetados al feto intraperitonealmente. Este método ha tenido relativo éxito.

Más recientemente, se han utilizado técnicas fetoscópicas para llegar a la vena umbilical en el sitio de inserción placentaria del cordón umbilical, bajo visión directa, por lo tanto permitiendo una transfusión intravascular directa con sangre $\mathrm{Rh}$ ne-

\footnotetext{
* Director Instituto ARGO

** Ginecólogo y obstetra. Práctica privada

*** Ginecólogo y obstetra. Hospital Central de la Policía Nacional
}

gativa (6). Esta técnica se torna más difícil después de la semana 30 , porque aumenta la relación del tamaño fetal versus el volumen del líquido, y también porque el líquido amniótico se torna opaco. La fetoscopia además requiere anestesia y al introducir un fetoscopio de gran calibre en el útero grávido, se corre un gran riesgo (7).

Posteriormente Bang. y Cols., reportan por primera vez y con éxito, la técnica de transfusión Intrauterina Intravascular, en fetos severamente isoinmunizados, bajo visualización directa con ultrasonido (8).

\section{Presentación del Caso}

Presentamos nuestra primera experiencia de Transfusión Intrauterina Intravascular de un feto severamente comprometido por isoinmunización Rh. El procedimiento se realizó en la Clínica del Country. Paciente de 33 años G3, P2, AO, quien se presentó a consulta por primera vez el 15 de enero de 1988, con 11 semanas de gestación, lo cual se confirmó con una ecografía. La paciente de grupo $\mathrm{O} \mathrm{Rh}$ negativo y el esposo grupo $\mathrm{O} \mathrm{RH}$ positivo. La historia obstétrica mostró lo siguiente:

En 1977 tuvo un parto vaginal normal, el producto, un recién nacido sano de sexo masculino, con $\mathrm{Rh}$ positivo. La madre nunca recibió el anti D (Rhesuman).

En 1985, tuvo otro parto vaginal normal a término. El producto, un recién nacido de sexo femenino $\mathrm{Rh}$ positivo, severamente isoinmunizado, que murió durante una exanguino-transfusión.

El embarazo actual fue de curso normal, excepto por la isoinmunización $\mathrm{Rh}$, que se evidenció 
por los títulos de Coombs indirectos (ver Tabla 1), y por el resultado de las amniocentesis seriadas que se hicieron para determinar el grado de hemólisis fetal (ver Tabla 2), mediante el análisis espectrofotométrico del líquido amniótico para la concentración de bilirrubina.

TABLA 1

\begin{tabular}{|lll|}
\hline Fecha & Edad Gestacional Título de Coombs \\
\hline Enero $8-88$ & 11.5 & $1 / 16$ \\
Marzo $17-88$ & 19.0 & $1 / 32$ \\
Abril 4-88 & 21.5 & $1 / 64$ \\
Abril 18-88 & 23.5 & $1 / 256$ \\
\hline
\end{tabular}

TABLA 2

\begin{tabular}{|lccc|}
\hline Fecha & Edad Gestacional DO 450 & L/S \\
\hline Abril 22-88 & 23.5 & 0.24 & - \\
Abril 29-88 & 24.5 & 0.25 & - \\
Mayo 11-88 & 26.0 & 0.25 & - \\
Mayo 25-88 & 28.0 & 0.18 & - \\
Junio 10-88 & 30.0 & 0.18 & 1.5 \\
\hline
\end{tabular}

Una ecografía practicada a las 30 semanas de gestación, mostró un feto con evidencia de ascitis moderada, edema subcutáneo, la placenta francamente hipertrófica (9). Ver foto No. 1. E1 perfil biofísico fetal dio un puntaje de 6 , es decir, una hipoactividad fetal como consecuencia de una hipoxia.

A esta edad gestacional se inyectó betametasona para inducir maduración pulmonar fetal.

$\mathrm{Al}$ analizar la curva de Liley (ver Figura 1), observamos que estábamos muy cerca de la zona III, hallazgo que sumado al de la ecografía y el Perfil Biofísico Fetal, nos llevó a la conclusión de que estábamos frente a un caso de un feto severamente hemolizado, con un riesgo extraordinario de morir in-útero y con inmadurez pulmonar. Por lo tanto, decidimos realizar una Transfusión Intrauterina Intravascular.

\section{Técnica}

La víspera del tratamiento se realizó una ecografía completa, con biometría fetal cuidadosa y con énfasis especial en la identificación y localización de la inserción del cordón en la placenta (Fo-

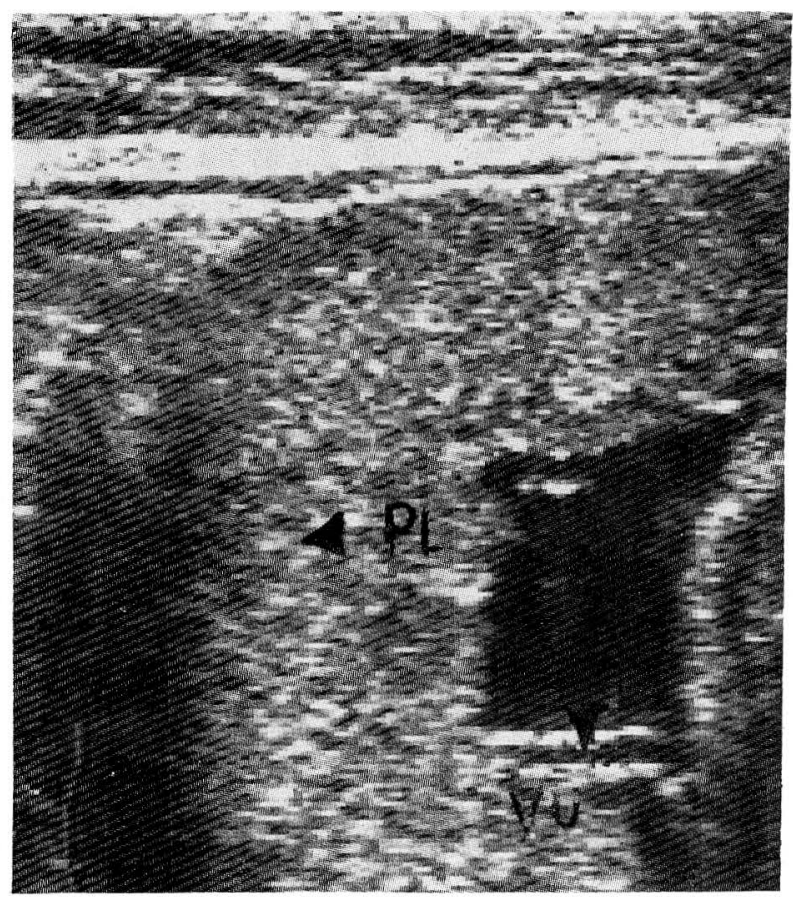

La punta de flecha con VU, indica la entrada de la vena umbilical en el sitio de la inserción placentario del cordón umbilical. La punta de flecha con PL, indica la placenta exageradamente hipertrófica.

to No. 1). El día de la intervención, la paciente fue hospitalizada, e inmediatamente antes de ser llevada a la sala de cirugía, se le tomó una muestra de sangre para determinar la Hemoglobina, el Hematocrito y el Volumen Corpuscular medio, con el objeto de tenerlos como referencia para compararlos con los obtenidos en la muestra fetal.

\section{FIGURA 1}

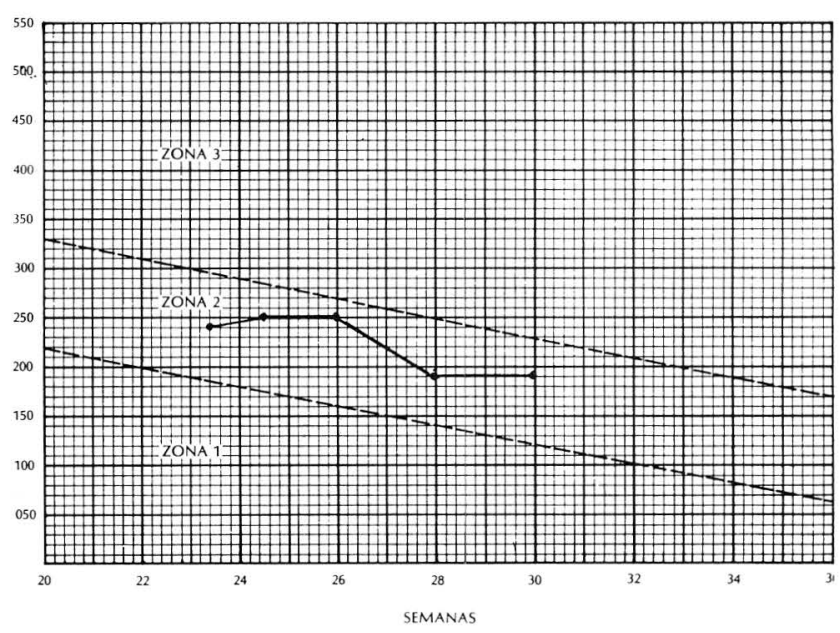

CURVA DE LILEY. Las determinaciones de la concentración de bilirrubina, muestra que estábamos muy cerca de la zona 3. 
Media hora antes del procedimiento, se le aplicaron $50 \mathrm{mg}$ de Meperidina por vía IM, y en el momento de cubrir a la paciente con los campos estériles, se le aplicaron por vía IV $10 \mathrm{mg}$ de Diazepán, $2.5 \mathrm{mg}$ de Terbutalina y $1 \mathrm{~g}$ de Ampicilina. Una vez preparado el abdomen de la paciente, utilizando un equipo de ultrasonido de tiempo real de alta resolución con un transductor lineal de $3.5 \mathrm{MHz}$ (Sonoline SL-Siemens), se localizó la inserción del cordón en la placenta. Bajo visión ecográfica directa, se procedió a puncionar la vena umbilical aproximadamente a $2 \mathrm{~cm}$ de la raíz del cordón, utilizando una aguja calibre 22 de $15 \mathrm{~cm}$. (Tipo Chiba); este objetivo se logró al segundo intento (Foto No. 2). Una vez insertada la aguja se obtuvo una muestra de sangre de $1 \mathrm{ml}$ que fue enviada al laboratorio para análisis, encontrándose: Hemoglobina 6.0\% Hematocrito 18 , Volumen Corpuscular medio 130, y RH Positivo (después del lavado de los glóbulos rojos). Confirmando por el V.C.M., y por el Rh, que se trataba de sangre fetal, se procedió a la inyección de

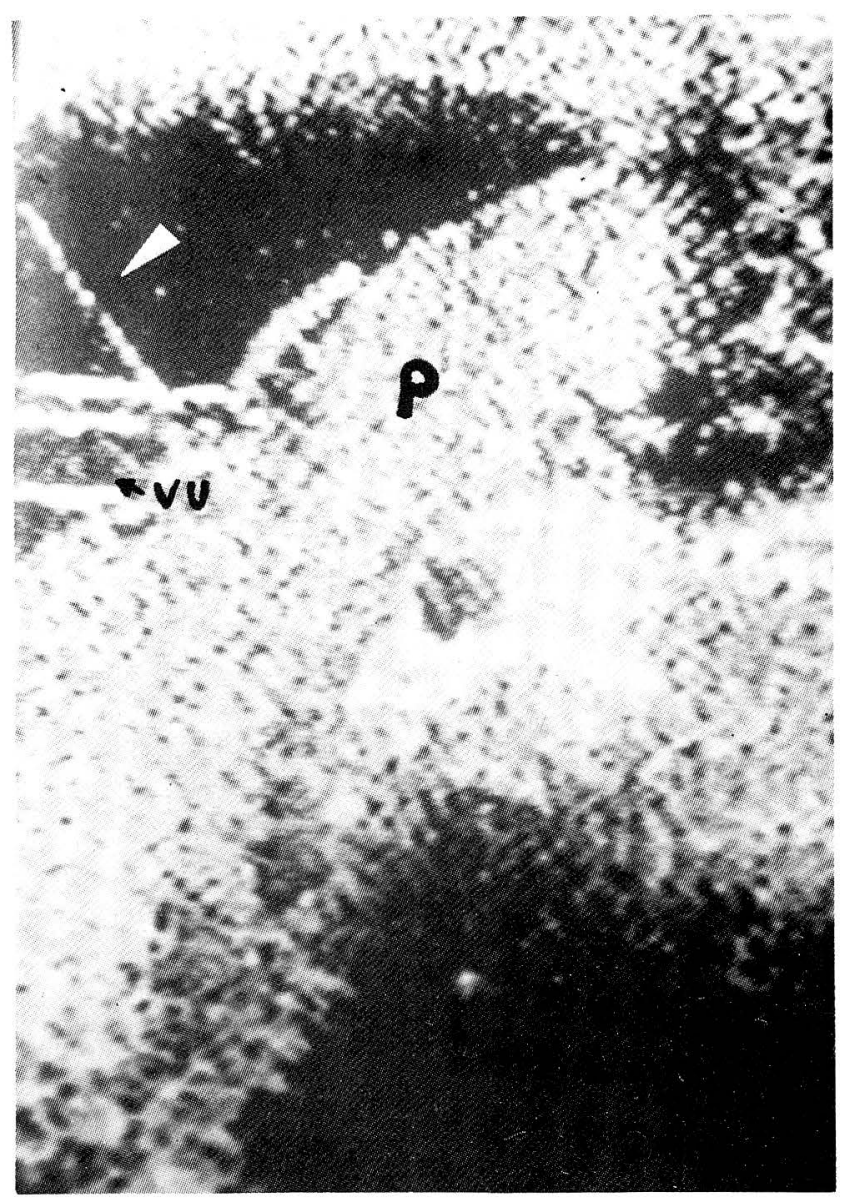

Se observa la localización de la aguja (punta de flecha), en la inserción placentaria del cordón. P: Placenta; VU: Vena Umbilical.

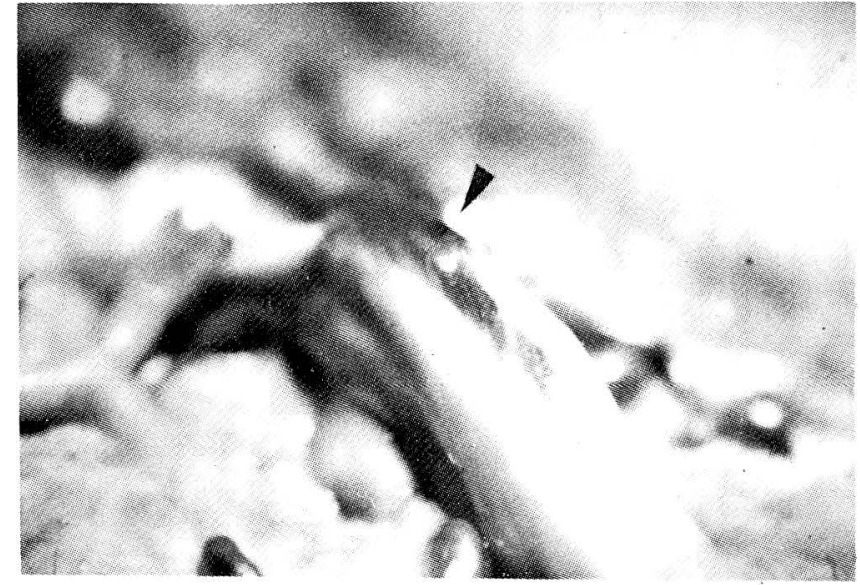

Pequeña equimosis (punta de flecha) en la gelatina de Wharton de la raíz del cordón umbilical.

$10 \mathrm{ml}$ de glóbulos rojos empaquetados, después de lo cual se tomó un nueva muestra para controlar la Hemoglobina y Hematocrito, pero durante la maniobra se desalojó la punta de la aguja, lo que obligó a relocalizarla para la inyección de los $8 \mathrm{ml}$ restantes, inyectándose un total de $18 \mathrm{ml}$.

Al finalizar la transfusión, la F.C.F. estaba en $160 \mathrm{lt} / \mathrm{min}$. y se observó un pequeño hematoma en la raíz del cordón, el cual se resolvió espontáneamente en 48 horas.

La paciente fue controlada con perfil biofísico tres veces por semana. Diez días después de la transfusión y una vez documentada la madurez pulmonar fetal por determinación de fosfatidil glicerol, se practicó una cesárea electiva obteniéndose un feto femenino de $1900 \mathrm{gm}$ de peso,con una hemoglobina en sangre de cordón de $5.7 \%$ que requirió 4 exanguinotransfusiones y fue dado de alta a los 10 días, luego de haber evolucionado en forma satisfactoria.

Al examen macroscópico de la placenta se observó una pequeña equimosis en la raíz del cordón, y una marca puntiforme en la gelatina de Wharton, la cual probablemente correspondía al sitio de la punción (Foto No. 3).

\section{DISCUSION}

Para que un procedimiento sea aceptado en la práctica clínica rutinaria, debe demostrar que sus beneficios sobrepasan las potenciales complicaciones. Aunque tan recientemente como en octubre de 1983, médicos de la autoridad del Dr. John 
Bowman desaconsejaban las técnicas de transfusión intrauterina intravascular (20), el transcurso de estos pocos años ha puesto de manifiesto las ventajas de esta técnica.

Desde la descripción hecha por Liley (10), la técnica de transfusión intrauterina había sufrido pocas y poco trascendentes modificaciones, y seguía representando un riesgo grande para el feto $(11,12)$. Sin embargo, la utilización de la ecografía como ayuda técnica en la realización del procedimiento $(4,13,14,15,16)$, permitió a los grupos con mayor experiencia como el de Winnipeg, disminuir a 0 la mortalidad asociada directamente con trauma fetal, y obtener una supervivencia del $100 \%$ en fetos no hidrópicos y del $75 \%$ en fetos hidrópicos (4). A pesar de esto, la transfusión intravascular puede tener varias ventajas:

1o. Disminuye el riesgo de trauma que representa la inserción de la aguja de transfusión en el abdomen fetal $(11,12)$.

2o. Los fetos hidrópicos pueden estar demasiado enfermos para absorber por vía linfática los glóbulos rojos depositados exitosamente en la cavidad peritoneal $(17,19,23)$.

3o. El acceso intravascular permite realizar la transfusión entre 4 y 6 semanas más temprano que por vía intraperitoneal, obteniendo así un $80 \%$ de supervivencia en los fetos menores de 25 semanas $(17)$.

4o. La transfusión intravascular, permite establecer la hemoclasificación fetal y evitar así transfusiones innecesarias; permite también determinar la hemoglobina y el hematocrito fetales, tanto pre como post transfusionales, facilitando el cálculo de la cantidad de sangre que debe transfundirse y permitiendo establecer la efectividad de la transfusión $(6,17,19,22)$.

5o. La determinación de la Hemoglobina y Hematocrito fetales durante el 2o. trimestre, permiten evaluar la severidad de la enfermedad fetal en forma más precisa que la curva de Liley (24).

Las primeras transfusiones intravasculares utilizando técnica fetoscópica, fueron reportadas por Rodeck en 1981. Aunque los resultados reportados son bastante buenos $(6,17)$, la fetoscopia, como ya anotamos, no deja dè presentar algunos inconvenientes: 1o. La técnica no es fácil y se requiere la amplia experiencia de Rodeck y sus colaboradores para lograr la cateterización de los vasos umbilicales; por lo tanto, es poco probable que en otros centros logren reproducirse sus excelentes resultados (23).

2o. La relación volumen amniótico/volumen fetal, disminuye a medida que progresa el embarazo, al tiempo que el líquido amniótico se va haciendo progresivamente turbio, especialmente cuando están aumentadas las bilirrubinas y demás pigmentos derivados de la hemoglobina $(18,23,25)$, lo cual dificulta considerablemente la fetoscopia alrededor de 30 semanas.

3o. La fetoscopia se asocia con un bajo pero significativo porcentaje de pérdidas fetales que oscila entre el 2 y el $5 \%$ según los distintos autores $(22,27,28,26,29)$.

El acceso percutáneo al sistema vascular fetal bajo control ecográfico, fue descrito inicialmente por Daffos, quien obtuvo sangre fetal para pruebas diagnósticas utilizando una aguja calibre 20 (28). En la revisión más numerosa de casos (25), este procedimiento se asoció solamente con un $1.1 \%$ y $0.8 \%$ de pérdidas fetales y abortos respectivamente. La posibilidad de usar la punción del cordón umbilical, como procedimiento terapéutico, también fue descrita por Daffos en el manejo de un feto con trombocitopenia autoinmune (30).

Bang, fue el pionero en tratar un feto severamente afectado por isoinmunización $\mathrm{Rh}$, mediante transfusión intravascular percutánea con control ecográfico, inyectando la sangre directamente en la vena umbilical intrahepática, utilizando una aguja No. 18 (8). Crespigny, reportó también éxito en 3 de 4 casos, utilizando una técnica similar (18). Sin embargo, el acceso a la vena umbilical en su porción intrahepática, presenta el riesgo de lesionar estructuras abdominales o de provocar una hemorragia intrahepática. Recientemente se ha descrito la transfusión intravascular, mediante la punción de la vena umbilical cerca de la inserción del cordón en la placenta, o en el abdomen fetal $(19,23,31)$, con lo cual se obtienen las ventajas de la transfusión intravascular con la simplicidad de la guía ecográfica, y evitando el riesgo que representa el acceso a la porción intra-abdominal de la vena umbilical.

Aún faltan por establecer parámetros definidos sobre el volumen de sangre que debe transfundirse, y el intervalo entre cada transfusión. Aunque 
algunos autores prefieren realizar una exanguinotransfusión (19), en un intento por disminuir el riesgo de sobrecarga cardíaca, la mayoría se limita a transfundir un volumen de glóbulos rojos calculado, para obtener un hematocrito fetal entre 30 y $40 \%$ $(6,17,18,23,8,31,32)$.

Estos valores de hematocrito, son los que se han encontrado en fetos sin enfermedad hemolíti$\mathrm{ca}$, en quienes se han hecho determinaciones de Hemoglobina y Hematocrito en sangre del cordón, durante el 2o. y 3er. trimestre (33).

Aunque se ha descrito la curarización del feto $(18,34)$ para la realización del procedimiento, consideramos que ésta no es necesaria en forma rutinaria, y que debe reservarse para aquellas situaciones en que por la localización de la inserción del cordón, la aguja debe atravesar un gran trayecto de la cavidad amniótica cerca de las extremidades o el tronco del feto, o deba realizarse la punción cerca de la inserción del cordón en el abdomen fetal.

En los demás casos, la sedación materna con Meperidina y Diazepán, produce una inmovilidad fetal suficiente para realizar el procedimiento con comodidad (32).

El caso que presentamos en este artículo, se trataba de un feto severamente afectado como lo hacian suponer la historia materna y las pruebas

\section{BIBLIOGRAFIA}

1. BOWMAN, J.M.: 'The management of Rh Isoinmunization. Obstet. Ginecol. 52: 1, 1978.

2. CLEWELL W.H., DUNNE M.G.; JOHNSON M.L. et al: Fetal Transfusion with real time ultrasound guidance. Obstet. Gynecol 57:516, 1981.

3. FRIGOLETTO, F.D.; UMANSKY, I.; BIRNHOLZ, J. et al : Intrauterine fetal transfusion in 365 fetuses during fifteen years. Am. J. obstet. Gynecol 139: 781, 1981.

4. HARMAN, C.R.; MANNING, F.A.; BOWMAN, J.M. et al: Severe $\mathrm{Rh}$ disease Poor outcome is inevitable. Am. J. Obstet. Gynecol 145: 823, 1983.

5. PEPPEREL, R.J.; COOPER, I.A.: Intense Antenatal Plasma Pheresis in severe rhesus isoinmunization. Aust NZ J. Obstet. Gynecol 18: 121, 1978.

6. RODECK, CH.; HOLAMN, C.A.; KARNICKI, J. Direct Intravascular fetal blood transfusion by fetoscopy in severe isoinmunization. Lancet 1: 625. 1981. biofísicas y de laboratorio; y como lo confirmaron las determinaciones de hemoglobina en sangre de cordón, tanto en el momento de la transfusión como en el nacimiento, así como la cantidad de exanguinotransfusiones que requirió después del parto. Gracias a la transfusión intrauterina, se logró aplazar el nacimiento hasta el momento en que presentaba madurez pulmonar suficiente para no agregar un sindrome de dificultad respiratoria al cuadro patológico, ya de por sí complicado, que presentaba este niño.

Resumiendo: La Transfusión Intravascular Inútero, representa un avance significativo en el manejo de isoinmunización por Rh. La utilización de la ecografía para guiar la introducción percutánea de la aguja en la circulación umbilical cerca de la raíz del cordón o del abdomen fetal, simplifica mucho el procedimiento. Aunque existen todavía algunas dificultades técnicas y se requiere de experiencia ecográfica, esta técnica ofrece grandes ventajas en el tratamiento de estos fetos severamente enfermos.

\section{RESUMEN}

Se presenta un caso de transfusión intravascular Intrauterina bajo control ecográfico, en un feto severamente afectado por isoinmunización $\mathrm{Rh}$. Las ventajas y los inconvenientes de las distintas técnicas de transfusión intrauterina son discutidas en este artículo.

7. RODECK, CH. Fetoscopy guided by real time ultrasound, for pure fetal blood samples, fetal skin samples, an examination of the fetus in utero. Br. J. Obstet. Gynecol 87: 449, 1980.

8. BANG, J.; BLOCK, J.E.; TROLLE, D. Ultrasound guided fetal intrauterine transfusions for severe rhesus hemolitic disease. Mr. Med. J. 1: 373, 1982.

9. BOWEN, F.W.; RENFIELD, M.: The detection of anti-D in Rh (D). negative Infants born of Rho (D)- positive mothers. Pediatr. Res. 10: 213, 1976.

10. LILEY, A.W.: Intrauterine transfusion of fetus in haemolytic disease. Br. Med. J. 2: 1107, 1963.

11. HAMILTON, E.G.: Intrauterine Transfusion: safeguard of peril. Am. J. Obst. Gynecol 50: 255, 1977.

12. PALMER, A.; GORDON, R.R.: A critical review of intrauterine fetal transfusion. Br. J. Obstet. Gynecol 83: 688, 1976.

13. HASHimoto, B.; FILLY, R.; CALlen, P.: Absorption of fetal intraperitoneal blood after intrauterine transfusion. $J$. Ultrasound Med. 6: 421, 1987.

14. BERKOWITZ, R.I.; HOBBINS, J.C.: Intrauterine Transfusion utilizing Ultrasound. Obstet. Gynecol 57: 33, 1981. 
15. FRIGOLETTO, F.D.; BIRNHOLZ, J.C.; ROTHCHILD, S.B Intrauterine Transfusion with the use of phased array ultrasonography: A new Technique. Am. J. Obstet. Gynecol 128: $239,1977$.

16. PLATT, L.D.; KEEGAN, K.A.; DRUZIN, M.L.: et al: Intrauterine Transfusion utilizing linear-array, real time B-scan: a preliminary report. Am. J. Obstet. Gynecol 135: 11 15, 1979.

17. RODECK, CH.; NICOLAIDES, K.W.; WARSOF, S.L. et al: The management of severe Rhesus isoinmunization by fetoscopic intravascular transfusions. Am. J. Obstet. Gynecol 150: $769,1984$.

18. CRESPIGNY, CH.; ROBINSON, H.P.; QUINN, M. et al: UItrasound guided fetal blood transfusion for severe Rhesus-isoinmunization. Obstet. Gynecol 66: 529, 1985.

19. GRANN, P.; COPEL, J.; PLAXE, S. et al: In-utero exchange transfusion by direct intravascular injection in severe erytroblastosis fetalis. N. Eng. J. Med. 314: 1431, 1986.

20. BOWMAN, J.M.; Blood group isoinmunization in obstetric practice. Curr. Probl. In Obstet and Gynecol. 7: 34, 1983.

21. BOWMAN, J.M.; MANNING, F.A.: Intrauterine fetal transfusions: Winnipeg 1982. Obstet. Gynecol 203: 61, 1983.

22. HOBBINS, J.C.; GRANNUN, P.; ROMERO, R.: Percutaneous umbilical blood samplig. Am. J. Obstet. Gynecol 152: 1, 1985.

23. BERKOWITZ, R.; CHITKARA, U.; GOLDBERG, J. et al: Intrauterine Intravascular transfusions for severe red blood cell isoinmunization: Ultrasound guided percutaneous approach. Am. J. Obstet. Gynecol 155: 574, 1986.

24. WARSOF, S.; NICOLAIDES, K.; RODECK, C.: Hidropesía Inmunitaria y no Inmunitaria. Clin. Obstet. Gynecol, 3: 678, 1986.

25. DAFFOS, F.; CAPELLA-PAULOVSKY, M.; FORESTIER, F. Fetal blood samplig during pregnancy with use of a needle guided by ultrasound: A study of 606 consecutive cases. Am. J. Obstet. Gynecol 153: 655, 1985.
26. Special Report: The status of fetoscopy and fetal tissue samplig. Prenat Diagn 4: 79, 1984.

27. RODECK, CH.: Obstetric Techniques in prenatal diagnosis. En: RODECK CH.; NICOLAIDES, K.H.; ed. Prenatal Diagnosis. London: Royal College of Obstet. and Gynecol. 15: 1984.

28. DAFFOS, F.; FORESTIER, F.; CAPELLA-PAVLOVSKI, $M$. Fetal blood sampling during the third trimester of pregnancy. Br. J. Obstet. Gynecol 91. $118,1984$.

29. DEVORE, GR.; VENUS, I.; HOBBINS, J.C.: Fetoscopy: general clinical approach. En: ROCKER, I.; LAWRENCE, K.M. eds. Fetoscopy. Amsterdam: Elsevier/North-Holland: 51, 1981.

30. DAfFos, F,; FORESTIER, F.; MULLER, J.Y. et al: Prenatal Treatment of alloinmune thrombo cytopenia. Lancet 2: 632,1984 .

31. BERKOWITZ, R.L.; CHITKARA, U.; GOLDERBERG, J.D.; et al: Intrauterine transfusion in-útero: the percutaneous approach. Am. J. Obstet. Gynecol. 154: 622, 1986.

32. BERKOWITZ, R.L.; CHITKARA, U.; WILKINS, I.; et al: Technical aspects intravascular intrauterine transfusions: Lessons learned from thirty-three. Am. J. obstet. Gynecol 157: 4, 1987.

33. NICOLAIDES, K.H.; RODECK, C.H.; MILLÄR, D.S. et al: Fetal Hematology in Rhesus Isoinmunization. Br. Med. J. 290: 661, 1981.

34. CRESPIGNY, C.H.; ROBINSON, H.P.; QUINN, M. et al: Curarisation of Fetus for intrauterine procedures. Lancet 1: 1164 1985. 